\title{
Quality and cost assessment of Canadian Urological Association microscopic hematuria guidelines in clinical practice: Turning urine into gold
}

\author{
Mark A. Assmus ${ }^{1}$, D. Beyer ${ }^{2}$; Joan Hanks ${ }^{3}$, Mathew Estey ${ }^{2,3}$; Keith F. Rourke ${ }^{1}$; Trevor \\ Schuler ${ }^{1}$; Tim Wollin ${ }^{1}$ \\ ${ }^{1}$ Department of Surgery, Division of Urology, Edmonton, AB, Canada; ${ }^{2}$ Department of Laboratory \\ Medicine and Pathology, Edmonton, AB, Canada; ${ }^{3}$ DynaLIFE Medical Laboratories, Edmonton, AB, \\ Canada
}

Cite as: Can Urol Assoc J 2019 February 7; Epub ahead of print. http://dx.doi.org/10.5489/cuaj.5809

Published online February 7, 2019

$* * *$

\section{Abstract}

Introduction: Asymptomatic microscopic hematuria (AMH) is defined in the Canadian Urological Association (CUA) guidelines as $>2$ red blood cells (RBCs) per high-powered field (hpf). Our objective is to evaluate guideline adherence for AMH at our centre. Secondarily, we aim to identify areas of the guideline that can be optimized.

Methods: We retrospectively reviewed 875 consecutive adults referred to two urologists for hematuria from June 2010-2016. Patient characteristics, risk factors, and outcomes were added to an encrypted REDCap database. Evaluation of microscopic hematuria reporting was performed by analyzing 681 urine samples reported as 1-5 RBC/hpf. Healthcare costs were obtained from Alberta Health Services (AHS), Data Integration and Management Repository (DIMR), and Alberta Society of Radiologists (ASR). Results: Of the 875 patients referred with hematuria, 400 had AMH. Overall, 96.5\% completed evaluation consistent with the CUA guideline. The incidence of pathology requiring surgical intervention was $21 / 400$ (5\%), with a $0.8 \%$ rate $(3 / 400)$ of urothelial cell carcinoma (UCC) (non-invasive, low-grade). No malignancy was found in nonsmokers with normal cytology, normal imaging and <50 RBC/hpf; $44 \%$ had AMH in the 1-5 RBCs/hpf range. Only 41\% (279/681) of urine samples categorized as 1-5 RBCs/hpf had guideline-defined microscopic hematuria. By changing local microscopic hematuria reporting to differentiate 1-2 and 3-5 RBCs/hpf, we estimate \$745 000 in annual savings. Conclusions: At our centre, CUA AMH guideline adherence is high. We did not find malignancy in non-smokers with normal cytology, imaging, and $<50 \mathrm{RBC} / \mathrm{hpf}$. We identified and changed regional microscopic hematuria reporting to fit the CUA 
definition, eliminating unnecessary investigations and healthcare costs.

\section{Introduction}

Microscopic hematuria ( $\mathrm{MH})$ is a common urinalysis abnormality, with a lifetime incidence of $6.5 \%$ in the general population ${ }^{1-5}$. Although there are a number of potential benign causes, ruling out genitourinary (GU) malignancies as the etiology for asymptomatic microscopic hematuria $(\mathrm{AMH})$ is paramount. In reviewing the literature, $\mathrm{MH}$ has been associated with a $0.5-10.5 \%$ rate of bladder cancer ${ }^{1-5}$. Despite the importance of evaluating the correct patients in a comprehensive manner, there remains a lack of consensus among international guidelines, as exemplified by contrasting the American Urological Association (AUA), Canadian Urological Association (CUA) and European guidelines for investigating hematuria.

In examining our current 2008 CUA guidelines, $\mathrm{MH}$ is defined as $>2 \mathrm{RBCs} / \mathrm{hpf}$ on two microscopic urinalysis (UA) without recent exercise, menses, sexual activity or instrumentation $^{6}$. Subsequent evaluation consists of a history, physical exam, urine cytology and upper tract imaging in the form of an ultrasound (US) or computed tomography (CT) with urogram phase. Cystoscopy is performed on patients $>40$ years old or with a positive/atypical urine cytology ${ }^{6}$. In patient's $\leq 40$ years old only those with risk factors for urothelial malignancy should proceed to cystoscopy ${ }^{6}$. Risk factors include smoking history, occupational chemical exposure, pelvic radiation, cyclophosphamide and storage symptoms.

In contrast, the 2008 British guidelines approach hematuria by defining either visible or non-visible hematuria on a fresh voided dipstick ${ }^{7}$. Again, the cut off of 40 years old or greater is utilized to dictate who requires cystoscopic evaluation.

In 2016, Bladder Cancer Canada (BCC), the Canadian Urologic Oncology Group (CUOG) and CUA published a consensus recommendation document to improve bladder cancer quality of care in Canada ${ }^{8}$. In that paper they reviewed the CUA MH guidelines with the following recommendations; urine cytology reserved for gross hematuria $(\mathrm{GH})$ or symptomatic hematuria, follow negative workup patients annually with UA and cytology x 3 years (If 2 consecutive normal UA then discontinue further workup), if $\mathrm{MH}$ persists or degree worsens then consider repeat evaluation every 3-5 years ${ }^{8}$. Also, similar to the 2012 AUA guideline update, the authors recommend decreasing the patient age cut off for those who require cystoscopy evaluation to $\geq 35$ years old irrespective of risk factors $^{8-9}$.

Of particular interest to our center, and despite a paucity of evidence, the guidelines that utilize urine microscopy in the definition of AMH unanimously use a cutoff of $>2 \mathrm{RBC} / \mathrm{hpf}$ to represent guideline defined amounts of microscopic blood in the 
urine. At the initiation of this study the Edmonton Zone reported urine hematuria microscopy ranges of: $0,1-5,6-10,11-25,26-50$ or $>50 \mathrm{RBC} / \mathrm{hpf}$. Due to these long standing reporting practices, remotely established Edmonton Zone Urology Referral Reference (EZURR) guidelines recommended that primary care physicians (PCP's) refer patients with $>5$ RBCs/hpf for MH evaluation. This difference in EZURR and CUA recommendations resulted in variable referring patterns, with some PCP clinics referring all patients with 1-5RBCs/hpf and other clinics only referring >5RBCs/hpf.

From the perspective of efficient and effective health care resource utilization, determining which patients need cystoscopic evaluation of the lower urinary tract (LUT) for $\mathrm{MH}$ is a crucial decision. At our centralized ambulatory Northern Alberta Urology Center (NAUC), approximately 2400 screening cystoscopies are performed annually for the investigation of $\mathrm{AMH}$, representing a substantial utilization of health care resources. Additionally, cystoscopy is an invasive investigation that is associated with significant patient anxiety ${ }^{10-12}$. Working within the Canadian universal health care model, a push for research and practice pattern changes such as the Choosing Wisely campaign prompted us to ensure our current guideline investigations are detecting clinically significant pathology (those requiring medical or surgical intervention or of importance to patients) in an efficient manner ${ }^{13-14}$.

The objective of this study was to examine our local practice for a cohort of adult patients referred for evaluation of $\mathrm{MH}$ and to identify the incidence of clinically significant pathology utilizing Canadian recommendations. We hoped to examine our adherence to the guidelines, ensure we were providing patients with the correct investigations and highlight potential quality improvement targets to minimize incomplete workups, loss to follow up or unnecessary health care expenses at our center.

\section{Methods}

\section{Study design}

After receiving institutional review board approval, we retrospectively reviewed 875 consecutive adults that were seen in urologic consultation for hematuria in Edmonton, AB, Canada by two urologists (KFR and TAW) between January 2010 - 2016. Of these 875 patients with hematuria, 400 had been referred for evaluation of AMH. Patient characteristics, hematuria history, UCC risk factors, investigations and outcomes were collected and managed using an encrypted Research Electronic Data Capture (REDCap) tool hosted at the University of Alberta.

\section{Outcome measures}

For our primary outcomes, we assessed detection of urothelial malignancy and adherence to guideline recommendations. Clinically significant urologic diagnoses included both malignant and non-malignant diagnoses that may require medical or surgical intervention 
or could be important to patients. We systematically reviewed all aspects of each patients AMH workup (history, physical, upper tract imaging, lower tract imaging, cystoscopy evaluation) and recorded the frequency of all malignant and non-malignant abnormalities detected (malignant tumours, benign cysts, benign tumours, congenital/anatomic abnormalities, urinary tract calculi, adrenal abnormalities, bladder outlet obstruction). Additionally, non-urologic incidentally detected abnormalities were recorded (peripheral vascular disease/aneurysms, uterine fibroids, ovarian cysts, bowel abnormalities). Secondary quality assessment (QA) outcomes involved analyzing 681 urine samples reported to have 1-5RBCs/hpf that underwent microscopy evaluation on three regional DynaLIFE Medical Labs analyzers over 2 consecutive days in the Edmonton Zone. Within the reporting category of 1-5RBs/hpf we identified the proportion of samples that had clinically significant hematuria ( $>2 \mathrm{RBCs} / \mathrm{hpf})$. Regional healthcare costs for the minimal CUA guideline recommended workup of AMH were obtained from Alberta Health Services (AHS), Alberta Medical Association (AMA), Data Integration and Management Repository (DIMR) and Alberta Society of Radiologists (ASR). We specified one confirmatory urinalysis, urine cytology, upper tract imaging via ultrasound of the kidneys, ureter \& bladder as well as urologic consultation with lower tract evaluation via cystoscopy in order to estimate the per patient cost of the minimal workup for AMH at our center.

\section{Statistical analysis}

Continuous variables are expressed as mean, median and range while proportions were used for categorical variables. GraphPad Prism (v6.0 Inc., La Jolla, California, USA) was used for our statistical analysis including one-way ANOVA analysis with Tukey's multiple comparisons test set to a significance of 0.05 (95\% CI).

\section{Results}

\section{Patient characteristics}

875 consecutive hematuria referrals seen by two urologists in Edmonton, Alberta from January 2010 - January 2016 were reviewed. 400/875 (46\%) had asymptomatic microscopic hematuria. 70 patients were excluded because they did not have microscopic or gross hematuria warranting a referral $(n=31)$, or they did not have reviewable data $(n=39)$. The remaining patients were referred for gross hematuria and not included in this analysis.

Table 1 outlines the patient characteristics of the 400 microscopic hematuria referrals. $262 / 400$ (66\%) patients were female with an overall mean age at consultation of 59 years old (range: 19-102 years old). There was no mean age difference at consultation between male (60 years old) and female (58 years old) patients ( $\mathrm{p}>0.05)$. 
Overall, 33/400 (8\%) of patients were $\leq 40$ years old with no difference between male and female patients (15 vs. 18 patients, $\mathrm{p}>0.05$ ).

The mean and median time from first positive urinalysis to consultation with urology was 34 and 11 months, respectively. There was no difference in median time to consultation between male and female patients (11 vs. 12 months, $\mathrm{p}>0.05$ ).

Urothelial Cell Carcinoma Risk Factors:

140/400 (35\%) identified smoking at least 1-pack year within the 10 years leading up to their consultation. 82/140 (59\%) of these patients actively smoked at the time of consultation. 6/400 (2\%) had additional urothelial cell carcinoma risk factors as outlined in Table 2.

\section{Urine dipstick/urinalysis}

Table 3 outlines the distribution of the maximal pre-consultation urine dipstick hemoglobin level. 5\% did not have hematuria detected on a reviewable urine dipstick prior to referral (including no dipstick performed, 0RBCs/hpf, trace hematuria/referring physician reported hematuria). The highest proportion of referrals had $1+$ hemoglobin on urine dipstick (45\%). Of note, $2 \%$ of patients were referred for microscopic hematuria with 4+ hemoglobin levels.

Table 4 contains the microscopic urinalysis results with $44 \%$ having 1-5RBCs/hpf as the highest degree of microscopic hematuria observed prior to consultation.

\section{Urine cytology}

Table 5 highlights our urine cytology results. 350/400 (88\%) had benign cytology, 32/400 (8\%) atypical, 18/400 (4\%) were not recorded and 0/400 (0\%) were malignant.

Of the 32 atypical urine cytology patients, imaging revealed 2 patients with benign renal cysts on ultrasound, 4 patients with non-obstructing renal calculi, 1 patient with a moderately elevated post void residual secondary to benign prostatic hyperplasia and 1 patient with an atrophic left renal moiety. Cystoscopy evaluation of these 32 patients with atypical urine cytology revealed 2 patients with bladder tumours, 3 patients with cystitis cystica, 1 patient with a bladder calculus, 1 patient with a urethral stricture and 2 patients with friable and trabeculated bladder walls. Final pathology on the 2 bladder tumours revealed one patient with low-grade superficial (Ta) UCC and the other with nonmalignant inflammatory changes.

\section{Genitourinary tract imaging}

333/400 (83\%) of patients underwent appropriate imaging prior to consultation. 85\% revealed benign imaging, with the $15 \%$ of detected upper tract and lower tract abnormalities outlined in Figure 1 and 2. 


\section{Cystoscopy}

383/400 (96\%) of patients underwent cystoscopy. Of the 17 patients who did not undergo cystoscopic workup, 6 patients did not meet CUA guideline recommendations for requiring a cystoscopy, 2 patients had no data recorded, 5 patients were no-shows and 4 patients refused the procedure.

Figure 3 highlights that 328/383 (86\%) of cystoscopies were normal. 5/383 (1\%) revealed a bladder tumour and 50/383 (13\%) had incidental non-malignant abnormalities identified as outlined in Figure 4, including the following etiologies potentially requiring surgery: bladder tumour, urethral stricture, bladder calculi and severe BPH.

\section{Bladder tumour detection}

5 bladder tumours were identified. 1/5 of these tumours were detected on pre-cystoscopy imaging in the form of unilateral hydronephrosis (final pathology = LG Ta UCC), with the remaining 4 having normal upper tract imaging. 3/5 (60\%) were malignant lowgrade superficial (Ta) UCC with the remaining 2 patients having non-malignant inflammatory findings. Table 6 breaks down the characteristics of the 5 patients with bladder tumours. Overall, comprehensive evaluation for AMH revealed 21/400 (5\%) patients requiring surgical intervention. $5 / 21$ underwent surgical intervention for bladder masses, 8/21 BOO/BPH/Urethral stricture, 4/21 bladder calculi, 3/21 ureteric calculi and 1 urachal mass/cyst.

\section{Guideline adherence}

21/400 (5\%) of microscopic hematuria workups identified significant pathology requiring further surgical intervention. 14/400 (3.5\%) of patients were $\leq 40$ years old with normal upper tracts, urine cytology, no UCC risk factors and asymptomatic microscopic hematuria. Deviating from the guidelines, 8/14 of these patients underwent cystoscopy, revealing one patient with cystitis cystica.

Urine Microscopy Reporting:

$41 \%$ (279/681) of urine samples categorized as having 1-5RBCs/hpf had guideline defined microscopic hematuria (>2RBCs/hpf) on final review, Figure 4.

\section{Cost savings}

With 44\% of MH referrals having 1-5RBCs/hpf and 41\% of these patients having guideline defined microscopic hematuria (>2RBCs/hpf), we can estimate that 103/400 patients underwent unnecessary $\mathrm{MH}$ comprehensive investigations and surgical consultation. Extrapolating these results to all patients referred to the NAUC seen by one of 15 adult staff urologists for $\mathrm{MH}$, roughly 620 patients are being referred and investigated unnecessarily on an annual basis. Utilizing costing data outlined in Table 7, the conservative regional cost of evaluating AMH is \$1196.85/patient. By changing the 
Edmonton Zone microscopic hematuria reporting into separate categories for 12RBCs/hpf and 3-5RBCs/hpf we estimate \$745,000 in annual health care savings, as seen in Figure 5.

\section{Discussion}

Overall, less than $1 \%$ of patients referred to our center for $\mathrm{MH}$ workup had a diagnosis of a malignancy (3/400). Although this incidence of detection falls within the range reported in the literature $(0.5-10.5 \%)^{1-5}$, by interrogating our regional $\mathrm{MH}$ laboratory-reporting ranges we detected that approximately $25 \%$ of patients that were evaluated did not have $\mathrm{MH}$ per the CUA guideline definition.

Despite our low rate of detecting cancer, referral to a urologist with comprehensive evaluation of the upper and lower tracts did detect multiple clinically significant non-malignant findings, some of which required further testing and surgical management. With $5 \%$ of referred patients having an abnormality that warrants surgical intervention and a large proportion initiating additional medical management, the value of comprehensively investigating this patient cohort was reinforced.

As noted above, when reviewing our preliminary data we identified that within the regional laboratory services in Edmonton, urine microscopy was not reported in a range that allowed identification of patients requiring $\mathrm{MH}$ workups per the CUA guidelines. Abnormal urine microscopy was reported if there was anywhere between 1-5 $\mathrm{RBCs} / \mathrm{hpf}$, including patients with $\leq 2 \mathrm{RBCs} / \mathrm{hpf}$. Once we identified that this potentially leads to a proportion of "MH" referrals for patients who only have 1-2RBCs/hpf and do not truly have $\mathrm{MH}$, we collaborated with DynaLIFE Medical Labs and the Department of Laboratory Medicine and Pathology to perform a quality assessment (QA) of UA reporting. This QA ultimately confirmed that a large proportion of UA reporting 15RBCs/hpf were not detecting guideline defined $\mathrm{MH}$. Using this data, we initiated a region wide change in urine microscopy reporting so that 1-2RBCs/hpf and 3-5RBCs/hpf were separately reported as of June 1, 2018.

By reporting the proportion of patients with guideline defined $\mathrm{MH}(>2 \mathrm{RBCs} / \mathrm{hpf})$ in the 1-5RBCs/hpf category we will prevent approximately 620 unnecessary comprehensive investigations for $\mathrm{MH}$ in our region per year. Including upper tract imaging, urine cytology, cystoscopy and specialist consultations (estimated $\$ 1196.85 /$ patient) this could translate into $\$ 745,000$ saved annually. This is a conservative estimate of savings since many patients will ultimately undergo many urine tests (urinalysis, urine cytology) along with additional imaging (CT urogram) as part of their AMH workup. We acknowledge that we are reporting savings from a health care payer perspective and did not take into account time off work and loss of potential earnings. Importantly, aside from cost savings, we can prevent numerous invasive procedures and psychological stress associated with a potentially unnecessary workup for 
$\mathrm{MH}$ in these patients. Alternatively, PCP's that previously followed EZURR referral guidelines (referral trigger of $>5 \mathrm{RBC} / \mathrm{hpf}$ ) now have the ability to correctly identify and refer patients with 3-5RBC/hpf. This will lead to an increase in appropriate comprehensive investigations for $\mathrm{MH}$.

One ongoing topic of discussion amongst the various $\mathrm{MH}$ guidelines is the age cut off for patients requiring lower tract cystoscopy (40 vs. 35 years old). In our study, very few patients $\leq 40$ years old were referred for $\mathrm{MH}$ evaluation (33 patients). Similar to a recent publication by Lippmann et al, the patients who had UCC detected in our cohort were older ( $>55$ years old) and had a history of smoking or abnormal imaging ${ }^{16}$. From the perspective of the recently published Canadian bladder cancer consensus paper, broadening the age range for who should proceed to cystoscopy only adds a small additional population/resource utilization pressure on the system, while ensuring that most bladder tumours are detected. If we examine our patient population that falls within this debated age range, only 14 patients were $\leq 40$ years old with normal upper tracts, urine cytology, no UCC risk factors and asymptomatic microscopic hematuria that did not have another indication for cystoscopy. 8/14 of these patients did proceed to cystoscopy evaluation without the detection of any clinically significant pathology. Per the current CUA guidelines these patients were subject to potentially unnecessary investigations and utilized additional health care resources.

Surprisingly, we did note a longer time from first positive MH UA to urology consultation than anticipated ( $>12$ months). In reviewing our data, a large portion of this stemmed from time between serial/confirmatory UA's and duration of time required to complete upper tract imaging. This may be in part due to the high volume nature of the NAUC, with its associated large catchment area. Many patients referred for urologic evaluation come from small northern Canada communities with barriers that may lead to delayed access to care (ex. CT-imaging). We expect this time to urologic consultation and cystoscopy evaluation to improve with our corrected UA MH reporting cut offs, which may decrease the annual MH referrals triaged at our center.

With respect to the urine cytology and cystoscopy results, only 32 patients had atypical urine cytology, with 1/32 having an ultimate diagnosis of a low grade UCC. This relatively inexpensive and easy to collect investigation may continue to have a screening/surveillance role for this patient population. Only $2.75 \%$ of patients referred for $\mathrm{MH}$ did not receive a cystoscopy when it was indicated, reinforcing excellent CUA guideline adherence at our center.

Study limitations include the retrospective nature of our chart review. Specific details on patient smoking history, risk factors and investigations prior to urology consultation were variably reported. Also, some patients seen at our centralized NAUC come from $>300 \mathrm{~km}$ away and may have laboratory investigations from our neighbouring 
provinces (British Columbia, Saskatchewan) that are not reviewable in this retrospective study. Additionally, we did not attempt to perform a comprehensive cost-benefit analysis for MH workup at our centre as the true global costs to the health care system extends beyond just the costs of each individual test and was not the intent of the study. In this study, we performed a QA of MH evaluation at our center and identified a cohort of patients that did not have clinically significant MH. For these patients, we determined the approximate investigative health care costs that were utilized in their evaluation.

Taken together, the results of this study have significant implications with respect to specialist responsibility to audit and perform ongoing QA assessments to ensure appropriate care and health care resources utilization. We identified that a simple and quick change in urine microscopy reporting within our region could result in fewer unnecessary investigations, cost savings and potential wait time improvements for patients that have indications for cystoscopy.

\section{Conclusion}

At our center, adherence to CUA MH guidelines appropriately screens and evaluates patients who require further intervention. We did not find any malignancy in nonsmokers with normal cytology, normal imaging and <50RBCs/hpf. By interrogating our local practices we identified and subsequently collaborated with DynaLIFE Medical Labs and the Department of Laboratory Medicine and Pathology to implement change in local $\mathrm{MH}$ reporting. The Edmonton Zone MH reporting cut off changes will significantly reduce unnecessary patient investigations and health care costs. 


\section{References}

1. Golin AL \& Howard RS. Asymptomatic microscopic hematuria. J Urol. 1980; 124:389-91.

2. Mohr DN, Offord KP, Owen RA, et al. Asymptomatic microhematuria and urologic disease: a population-based study. JAMA 1986; 256:224-9.

3. Sultana SR, Goodman CM, Byrne DJ, et al. Microscopic hematuria: urological investigation using a standard protocol. Br J Urol. 1996; 78:691-8.

4. Khadra MH, Pickard RS, Charlton M, et al. A prospective analysis of 1,930 patients with hematuria to evaluate current diagnostic practice. J Urol. 2000; 163:524-7.

5. Jung H, Gleason JM, Loo RK, et al. Association of hematuria on microscopic urinalysis and risk of urinary tract cancer. J Urol. 2011; 185:1698-70. http://dx.doi.org/10.1016/j.juro.2010.12.093

6. Wollin T, Laroche B, Psooy K. Canadian guidelines for the management of asymptomatic microscopic hematuria in adults. Can Urol Assoc J. 2009; 3:77-80.

7. Anderson J, Fawcett D, Feehally J, et al. Joint Consensus Statement on the Initial Assessment of Haematuira. July 2008. BAUS/RA Guidelines.

8. Kassouf W, Aprikian A, Black P, et al. Recommendations for the improvement of bladder cancer quality of care in Canada: A consensus document reviewed and endorsed by Bladder Cancer Canada (BCC), Canadian Urologic Oncology Group (CUOG), and Canadian Urological Association (CUA), December 2015. Can Urol Assoc J. 2016; 10(1-2):E46-80. http://dx.doi.org/10.5489/cuaj.3583

9. Davis R, Jones JS, Barocas DA, et al. Diagnosis, evaluation and follow-up of asymptomatic microhematuria (AMH) in adults: AUA guideline 2012. Unabridged on-line version, available at www.auanet.org. Accessed Dec 30, 2017.

10. Biardeau X, Lam O, Van Ba, et al. Prospective evaluation of anxiety, pain and embarrassment associated with cystoscopy and urodynamic testing in clinical practice. Can Urol Assoc J. 2017; 11(3-4): 104-10. http://dx.doi.org/10.5489/cuaj.4127

11. Ellis G, Pridgeon S, Lamb BW, et al. Psychological distress in out-patients undergoing flexible cystoscopy for the investigations of bladder cancer. $J$ of Clinical Urology. 2015; Vol 8(3) 196-201. doi:10.1177/2051415814551821

12. Raheem OA, Mirheydar HS, Lee HJ, et al. Does listening to music during officebased flexible cystoscopy decrease anxiety in patients; a prospective randomized trial. J of Endo. 2017. Vol 29(7) 791-796. doi:10.1089/end.2015.0029

13. American Board of Internal Medicine Foundation [press release] Choosing Wisely: five things physicians and patients should question. Washington, DC: American Board of Internal Medicine Foundation; 2012. Accessed July 1, 2018 from: www.choosingwisely.org/choosing-wisely-five-things-physicians-andpatients-should-question-press-release-april-4-2012/. 
14. Canadian Medical Association [website] Choosing Wisely Canada. Ottawa, ON: Canadian Medical Association; 2014. Accessed June 10, 2018 from: www.cma.ca/En/Pages/choosing-wisely-canada.aspx.

15. Harris PA, Taylor R, Thielke R, et al. Research electronic data capture (REDCap) - A metadata-driven methodology and workflow process for providing translational research informatics support. J Biomed Inform. 2009 Apr;42(2):37781

16. Lippmann QK, Slezak JM, Menefee SA, et al. Evaluation of microscopic hematuria and risk of urologic cancer in female patients. Am. J. Obstet. Gynecol. February 2017; 216:146.e1-7. 


\section{Figures and Tables}

Fig. 1. Frequency of abnormalities detected on upper tract imaging for $\mathrm{MH}$ evaluation. AAA: abdominal aortic aneurysm; AML: angiomyolipoma; GU: genito-urinary.

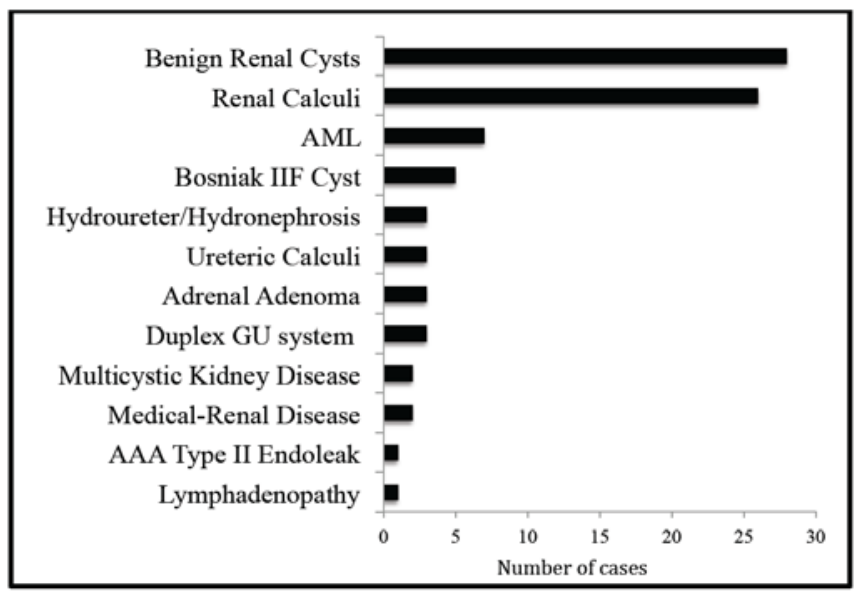

Fig. 2. Frequency of abnormalities detected on lower tract imaging for $\mathrm{MH}$ evaluation. BPH: benign prostatic hyperplasia; PVR: post-void residual; SBO: small bowel obstruction.

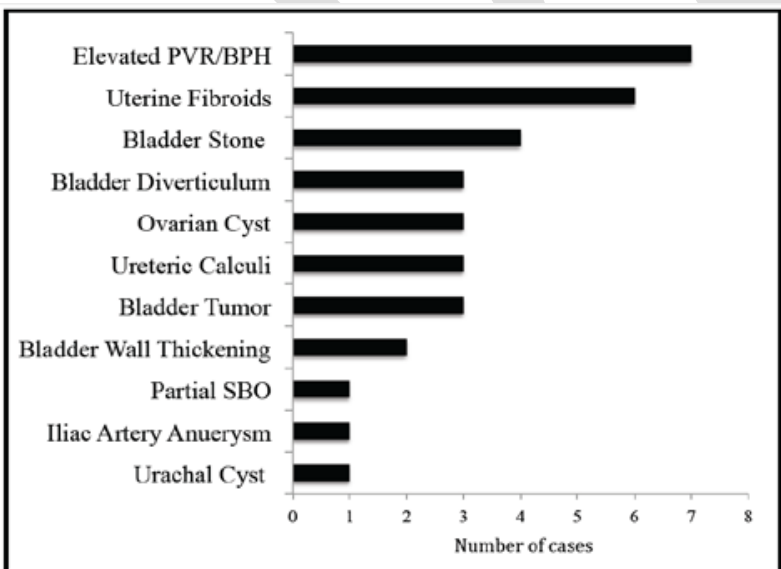


Fig. 3. Results for $383 \mathrm{MH}$ patients that proceeded to cystoscopic evaluation.

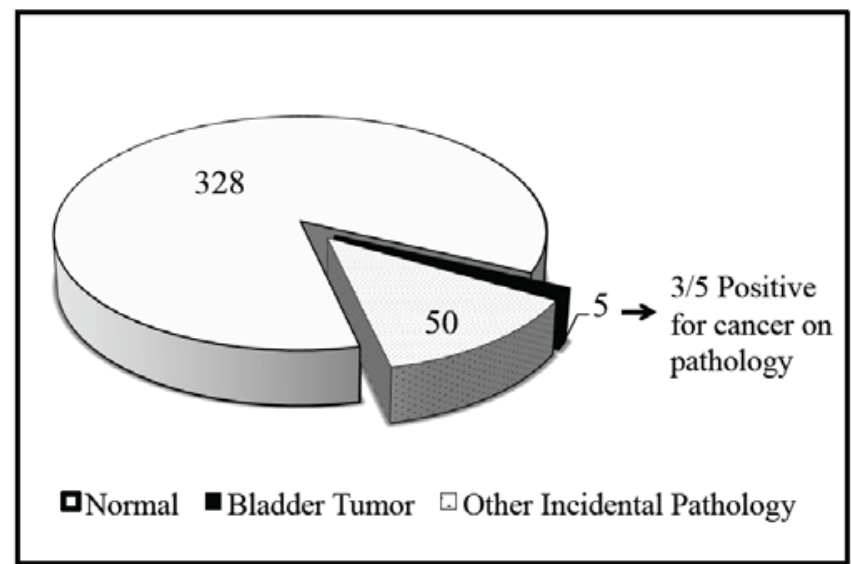

Fig. 4. Proportion of guideline defined ( $>2 \mathrm{RBCs} / \mathrm{hpf}$ ) $\mathrm{MH}$ on 681 consecutive urine microscopies that were initially reported as $1-5 \mathrm{RBCs} / \mathrm{hpf}$.

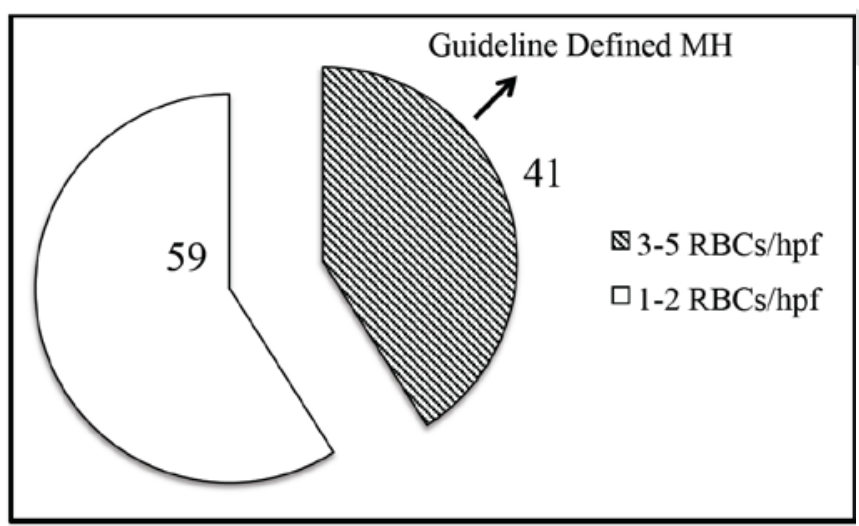


Fig. 5. Predicted patient care and healthcare resource benefit of implementing differentiated reporting ranges for $\mathrm{MH}$ in the Edmonton zone (0-2 RBCs/hpf and 3-5 RBCs/hpf).

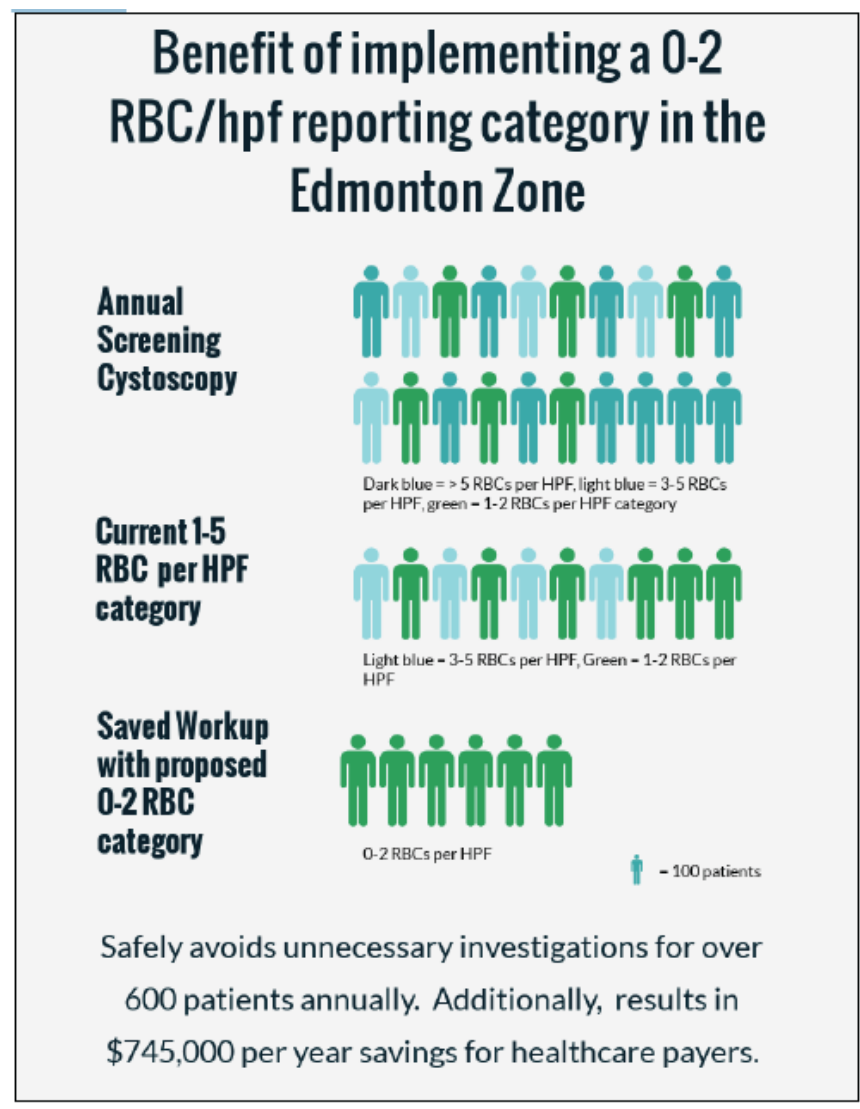




\begin{tabular}{|c|c|c|c|}
\hline Characteristic & Male & Female & Total \\
\hline Patients, n (\%) & $\begin{array}{l}138 \\
(34)\end{array}$ & $\begin{array}{l}262 \\
(66)\end{array}$ & $\begin{array}{c}400 \\
(100)\end{array}$ \\
\hline Mean age at consult, years (range) & $\begin{array}{c}60 \\
(19-102)\end{array}$ & $\begin{array}{c}58 \\
(24-91)\end{array}$ & $\begin{array}{c}59 \\
(19-102)\end{array}$ \\
\hline$\leq 40$ years old, $\mathrm{n}(\%)$ & $\begin{array}{c}15 \\
(11)\end{array}$ & $\begin{array}{l}18 \\
(7)\end{array}$ & $\begin{array}{l}33 \\
(8)\end{array}$ \\
\hline$>40$ years old, $n(\%)$ & $\begin{array}{l}123 \\
(89)\end{array}$ & $\begin{array}{l}244 \\
(93)\end{array}$ & $\begin{array}{l}367 \\
(92)\end{array}$ \\
\hline $\begin{array}{l}\text { Average time (months) from }+\mathrm{UA} \text { to consult } \\
\text { (mean/median) }\end{array}$ & $30 / 11$ & $37 / 12$ & $34 / 11$ \\
\hline Range time (months) from +UA to consult & $1-168$ & $1-300$ & $1-300$ \\
\hline
\end{tabular}

UA: urinalysis.

\begin{tabular}{|l|c|c|}
\hline Table 2. Bladder cancer risk factors for our patient cohort \\
\hline Bladder cancer risk factor & Number & \% \\
\hline Smoked within the last 10 years & 140 & 35 \\
Current smoker at time of cystoscopy & 82 & 21 \\
\hline Other risk factors & 6 & 1.5 \\
\hline Pelvic radiation & 2 & 0.5 \\
\hline Heavy petroleum exposure & 2 & 0.5 \\
\hline Cyclophosphamide & 1 & 0.25 \\
\hline Dye/textile industry & 1 & 0.25 \\
\hline
\end{tabular}




\begin{tabular}{|l|c|}
\hline $\begin{array}{l}\text { Table 3. Maximal urine dipstick hematuria level reviewable } \\
\text { prior to urology consultation }\end{array}$ \\
\hline Pre-consultation urine dipstick & \% patients \\
\hline None/trace & 5 \\
\hline $1+$ & 45 \\
\hline $2+$ & 34 \\
\hline $3+$ & 14 \\
\hline $4+$ & 2 \\
\hline
\end{tabular}

\begin{tabular}{|l|c|}
$\begin{array}{l}\text { Table 4. Maximal urine microscopy hematuria level } \\
\text { reviewable prior to urology consultation }\end{array}$ \\
\hline $\begin{array}{l}\text { Pre-consultation microscopic urinalysis } \\
\text { (RBCs/hpf) }\end{array}$ & \% patients \\
\hline $1-5$ & 44 \\
\hline $6-10$ & 19 \\
\hline $11-25$ & 21 \\
\hline $26-50$ & 6 \\
\hline$>50$ & 10 \\
\hline
\end{tabular}

\begin{tabular}{|l|c|}
$\begin{array}{l}\text { Table 5: Cytology outcomes for } 400 \text { patients referred for } \\
\text { MH evaluation }\end{array}$ & $\begin{array}{c}\text { Number (\%) } \\
\text { of patients }\end{array}$ \\
\hline Cytology result & $350(88)$ \\
\hline Benign & $32(8)$ \\
\hline Atypical & $0(0)$ \\
\hline Malignant & $18(4)$ \\
\hline Not recorded/reviewable at consult & \\
\hline
\end{tabular}




\begin{tabular}{|c|c|c|c|c|c|c|c|}
\hline $\begin{array}{l}\text { Age/ } \\
\text { Sex }\end{array}$ & $\begin{array}{c}\text { Max } \\
\text { UA } \\
\text { (RBCs/ } \\
\text { hpf) }\end{array}$ & $\begin{array}{c}\text { AMH } \\
\text { duration } \\
\text { (months) }\end{array}$ & $\begin{array}{c}\text { Smoker } \\
(\mathrm{Y} / \mathrm{N})\end{array}$ & Cytology & Imaging & Cysto results & Pathology \\
\hline $66 / F$ & $1-5$ & 5 & $\mathrm{~N}$ & Benign & $\begin{array}{l}\text { Unilateral } \\
\text { hydro }\end{array}$ & $\begin{array}{l}\text { Left UO and } \\
\text { dome polyp }\end{array}$ & LG-Ta UCC \\
\hline $58 / F$ & $>50$ & 6 & $\mathrm{~N}$ & Benign & Normal & Polyp & LG-Ta UCC \\
\hline $67 / F$ & $26-50$ & 90 & $\mathrm{~N}$ & Atypical & Normal & $\begin{array}{c}\text { Right UO } \\
\text { mucosal lesion }\end{array}$ & Non-malignant \\
\hline 77/M & $11-25$ & 142 & $\mathrm{Y}$ & Unknown & Normal & Polyp & LG-Ta UCC \\
\hline $51 / \mathrm{F}$ & $11-25$ & 4 & $\mathrm{Y}$ & Benign & Normal & $\begin{array}{l}\text { Inflammatory/ } \\
\text { edematous } \\
\text { patches }\end{array}$ & Non-malignant \\
\hline
\end{tabular}

F: female; M: male; UA: urinalysis; UCC: urothelial cell carcinoma.

\begin{tabular}{|l|c|}
\hline \multicolumn{2}{|l|}{$\begin{array}{l}\text { Table 7. Local cost of base investigations recommended by CUA } \\
\text { guidelines for AMH }\end{array}$} \\
\hline Investigation & Cost (\$) \\
\hline Urinalysis + microscopy & 32.03 \\
\hline Urine cytology & 359.00 \\
\hline $\begin{array}{l}\text { Surgical specialist clinical consultation } \\
\text { (Comprehensive AHS clinic facility costs) }\end{array}$ & 173.00 \\
\hline $\begin{array}{l}\text { Upper tract imaging evaluation (radiologist \& } \\
\text { ultrasound - kidney, ureter, bladder) }\end{array}$ & 447.00 \\
\hline Lower tract procedural evaluation (cystoscopy) & 178.00 \\
\hline Urologist & $\mathbf{- 9 3 . 0 0}$ \\
\hline $\begin{array}{l}\text { AMH consultation } \\
\text { Cystoscopy performed }\end{array}$ & $\mathbf{- 8 5 . 0 0}$ \\
\hline Total & $\mathbf{1 1 9 6 . 8 5}$ \\
\hline
\end{tabular}

AHS: Alberta Health Services; AMH: asymptomatic microscopic hematuria; CUA: Canadian Urological association. 